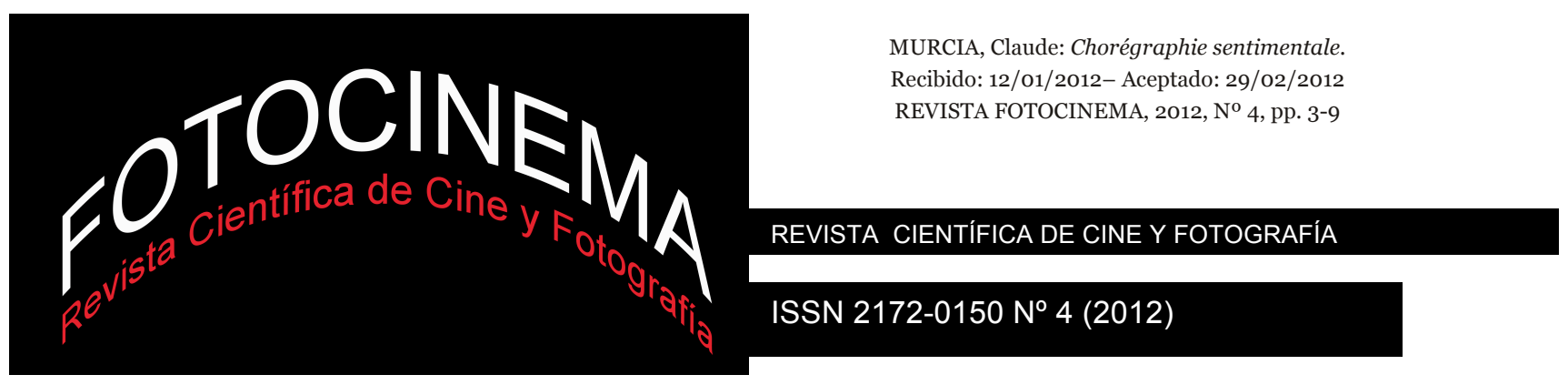

\title{
CHORÉGRAPHIE SENTIMENTALE
}

\section{COREOGRAFÍA SENTIMENTAL}

\section{Claude Murcia}

Université Diderot-Paris 7

\section{Resumen:}

$S$ a g i t a r i o (2001), film à la fois enjoué et mélancolique, présente une structure «mosaïque» qui substitue à la hiérarchisation des événements selon une logique causale la restitution d'un «état du monde». L'arbitraire de la contiguïté spatiale se joint à l'ubiquité énonciative pour construire un monde dominé par la discontinuité et l'inconstance. A l'éclatement structurel et à la dispersion du récit correspond la vision d'un sujet changeant et multiple qui semble mettre en échec tout principe de continuité malgré le recours -qu'on peut penser plaisamment générique- au «happy ending».

\begin{abstract}
:
Sagitario (2001) de Vicente Molina, película a la vez alegre y melancólica, presenta una estructura «mosaica» que frente a la jerarquización de los hechos según una lógica causal, se sustituye por un «estado del mundo». La arbitrariedad de la contigüidad espacial se une a la ubicuidad enunciativa para construir un mundo dominado por la discontinuidad y la inconstancia. A la ruptura estructural y a la dispersión del relato corresponde la visión de un sujeto cambiante $y$ múltiple que parece derrotar todo principio de continuidad a pesar del recurso -que puede uno suponer lúdicamente genérico- al «happy end».
\end{abstract}

\section{Palabras clave:}

Vicente Molina Foix; Sagitario; narración; posmoderno.

\section{Key words:}

Vicente Molina Foix; Sagitario; narration; postmoderne. 
Aucun ne fait certain dessain de sa vie, et n'en délibérons qu'à parcelles. (...) Nous sommes tous de lopins et d'une contexture si informe et diverse, que chaque pièce, chaque moment faict son jeu.

Montaigne

\section{Introducción}

En 2001, Vicente Molina Foix nous surprend par un premier film -Sagitario-, à la fois tendre, drôle et mélancolique. Le film offre sans aucun doute la lisibilité d'une structure narrative linéaire qui n'est parasitée par aucune anachronie. Cet effet de continuité -visible dans la succession annoncée des jours puis des mois- se trouve toutefois rapidement brouillé par diverses stratégies d'éclatement qui tendent à défaire les liens logiques de la causalité sur lesquels se fondent la narration conventionnelle. «A DISTANCE RESPECTUEUSE DE L'ORDRE ET DU DÉSORDRE» (Bresson, 1975: 59), le récit privilégie l'aléatoire au détriment de la nécessité en s'en remettant la plupart du temps aux forces du hasard, ou de quelque chose qui y ressemble. Car modeler la syntaxe narrative sur la chronologie calendaire, c'est détrôner les connexions logiques au profit d'un arbitraire ouvert à tous les possibles. La

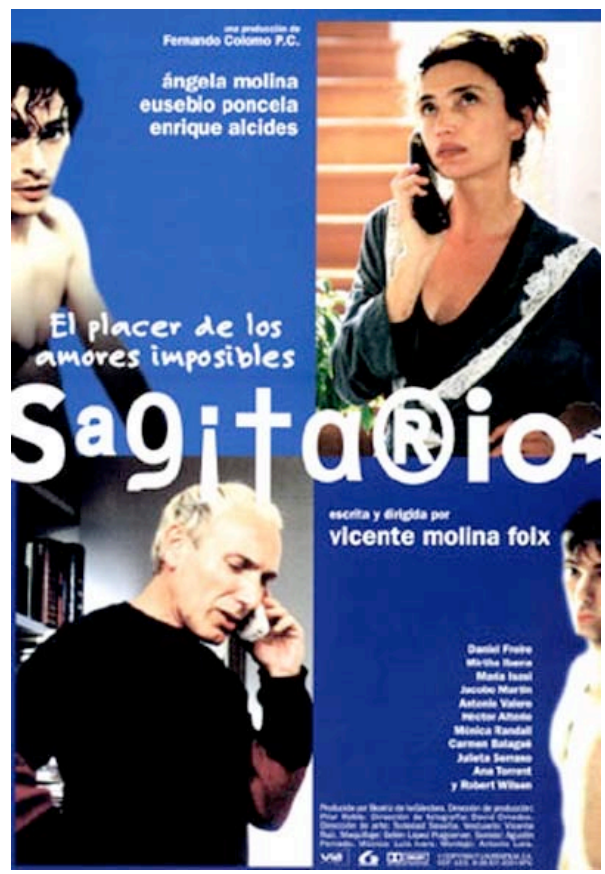
journée comme temps de référence, comme le précise Lucien Dällenbach, «est la durée la mieux accordée à la restitution esthétique de l'état du monde. Or, c'est cette restitution spécifique qui constitue, à l'exclusion de tout, la justification de l'art et de la littérature» (Dällenbach, 2001: 148). C'est bien cet état du monde, ces instants de vie saisis dans leur pur présent, que cherche à restituer Sagitario, coupés d'une totalité qui leur donnerait une cohérence et un sens rassurants. Le privilège de la journée, ajoute Dällenbach, «réside dans le support optimal qu'elle apporte à une esthétique et à une poétique de la 
diversité» (Dällenbach, 2001: 150). Car la plasticité de la journée comme modèle de temporalité narrative légitime l'ubiquité de l'énonciation, qui passe ainsi d'un personnage à un autre, d'un lieu à un autre, dans une alternance que le jour de la semaine suffit à motiver. D'une façon analogue, le choix d'explorer successivement les différentes parcelles d'un même espace dans lequel sont rassemblés les personnages distend les liens de causalité pour leur préférer l'idée de contingence. C'est le cas de cet immeuble (on pense à Hitchcock) ${ }^{1}$ dans lequel vivent la voyante, Juan et sa famille, Ana et sa belle-mère auxquelles vient s'agréger Gustavo, à qui Rosa viendra rendre visite... L'arbitraire de la conguïté spatiale vient alors se substituer aux contraintes de l'enchaînement causal.

Un principe du même ordre préside aux «digressions» de la caméra qui, suivant un personnage qui en croise un autre, quitte le premier pour s'attacher au second, semblant se soumettre au seul caprice d'une énonciation soudain attirée par un nouvel objet... C'est notamment le cas lorsque la caméra, qui suivait Juan en moto, l'abandonne quand il passe derrière Darío et Gustavo attablés à la terrasse du café pour rester avec eux; ou encore lorsque la caméra lâche Rafael dans sa course pour suivre l'homme (son gourou) qu'il vient de croiser sans le voir.

L'élasticité, la souplesse d'une telle structure, capable d'accueillir tous les possibles narratifs, s'ouvre «naturellement» aux hasards de la rencontre, motif central de la diégèse. La multiplicité des personnages s'accorde à l'absence de vectorisation du récit et à son aspect peu contraint, formant un film «choral» dont la polyfocalisation légitime la «dispersion» du récit. Tout au plus peut-on parler d'un couple central -Rosa/Jaime- autour duquel gravite tous les autres personnages dans un effet chorégraphique qui n'annule pas pour autant les singularités. Le montage «alterné» entre les différents personnages qui se croisent et se rencontrent au gré des circonstances (on pense à Altman) construit un récit fondé davantage sur la juxtaposition que sur la hiérarchisation de ses éléments, dans un effet «démocratique» qui inscrit le film dans une contemporanéité très actuelle.

\footnotetext{
1. A ce propos, l'apparition fugitive de Vicente Molina à la sortie du Cinestudio est-il un effet de signature à la Hitchcock?
} 
Le titre du film -aussitôt développé par le premier plan- inscrit d'emblée la fiction sous le signe d'une transcendance, ou d'un Hasard (les petites annonces prendront plus tard le relais de la voyance), à quoi les personnages semblent s'en remettre pour décider de leurs amours et de leur destinée.

Toutes ces stratégies convergent vers la dispersion et l'éclatement dont rend compte exemplairement le début du film: Lundi. Un premier plan fait apparaître une emblématique boule de cristal puis la caméra, glissant le long de la façade de l'immeuble, s'introduit, dans la contiguïté spatiale et syntagmatique, dans l'appartement de Juan et de sa famille. Coupe franche. Dans un bar, Juan s'accoude aux côtés de Gustavo dont il fait ainsi la connaissance et qui, en sortant, passe devant «el hombre destinado» sur lequel la caméra s'arrête. Le désir de ce dernier fait naitre brièvement, en surimpression optique, le visage de Rosa qui lâche au même instant un pépiement insolite. Coupe franche. Juan à moto va livrer des pizzas. Coupe franche. Plan d'une plume assortie du même pépiement, suivi d'un plan d'ensemble de personnages -dont Rosa- jouant à deviner des titres de films (le premier, d'Hitchcock...). Juan, sur ces entrefaites, arrive avec la pizza et s'attarde quelque peu. Rosa et Jaime semblent le trouver à leur goût.

On le voit, ce qui régit ici l'(dés)ordre de la narration a partie liée avec le discontinu et le multiple plutôt qu'avec la continuité d'une vision homogène, hiérarchisée selon les lois d'une logique rationalisante.

Ce faisant, l'énonciation dénonce plaisamment les contraintes de la narrativité tout en s'y dérobant. Une démarche analogue se retrouve chez certains écrivains
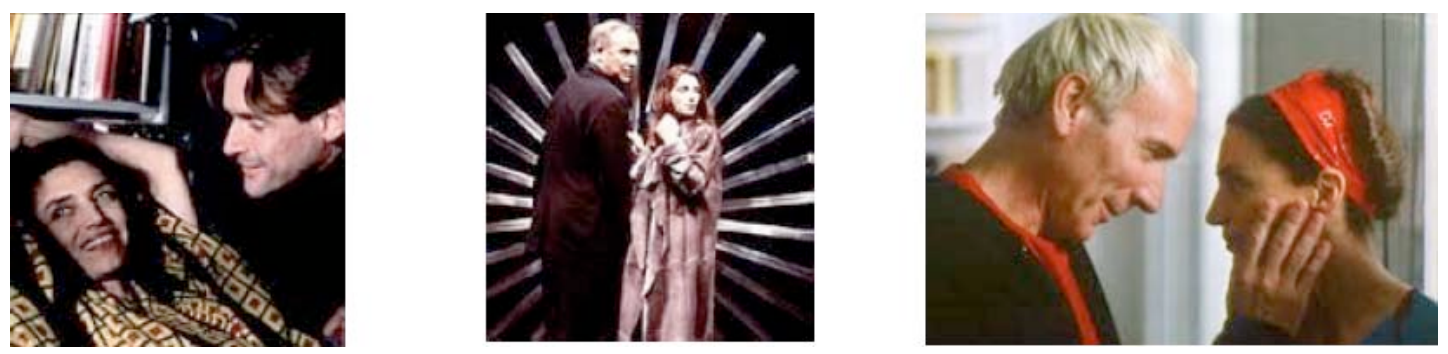

comme Jean Echenoz, qui se plaît à exhiber avec humour l'arbitraire de la narration: "Comme il s'endormait, la soupape d'une cocote-minute se déplaça légèrement dans la cuisine du troisième gauche au 118 rue Amelot, à six stations de métro de là...» (Echenoz, 1983 : 132). 
Une telle structure éclatée, polycentrée, hétérogène, capricieuse, discontinue apparaît comme le corrélat formel d'une vision de l'être humain comme sujet lui-même multiple et discontinu, dont l'unité identitaire s'avère problématique. Trouble identitaire que même le statut professionnel, garant d'une certaine stabilité, a du mal à masquer. Ainsi, l'échec semble obséder Darío qui, de «grand professeur de la faculté d'architecture» est devenu scénographe et se consacre à construire des décors en carton-pâte. Notion d'échec immédiatement relativisée par son disciple Gustavo. Rosa, peintre, est en mal d’inspiration et Jaime, comédien, après avoir fait le clown dans le café-théâtre et déclenché l'enthousiasme, s'éloigne seul et mélancolique, le Misteri d'Elx (on pense à Saura) ${ }^{2}$ magnifiant sa tristesse. L'individu apparaît le plus souvent comme un être contradictoire et changeant. Rosa oscille entre trois hommes, le seul qu'elle aime vraiment -Jaime- étant «clínicamente maricón». Séduite par Gustavo qu'elle vient de rencontrer, elle s'en détourne bientôt, déçue par son égoisme. Attirée un moment par son ex-mari rentré d'Afrique -Tarzan, comme le surnomme ironiquement Jaime-, elle cède un temps aux charmes juvéniles du «burbujeante» Juan dont l'inculture («Sorna, Juan, sorna») finit bien vite par l'insupporter. Signe, d'ailleurs, d'une société qui, malgré son ouverture et son métissage, a du mal à accepter les transgressions: Juan, accepté d'abord -par Rosa (dans les faits) et par Jaime (dans le discours) - avec enthousiasme, ne parvient pas à dépasser le statut d'objet sexuel. Jaime, contre toute attente (de sa part), semble davantage prêt à intégrer cet «autre» social qu'est Rafa. La contradiction, chez lui, est ailleurs: lui qui, naguère, rationalisait sa relation avec Darío, la considérait avec une lucidité critique, proclamait avec un certain cynisme l'inexistence de l'amour, s'effondre quand il se voit abandonné sans explication par le jeune Rafael. Rosa, toujours prête à adopter les jugements de Jaime, pour qui hier encore les sagittaire étaient amoureux et fidèles, demeure perplexe et désarmée devant un tel revirement. Cette ronde des sentiments (on pense à Ophüls), cette incapacité à aimer durablement ou à aimer autre chose que le désir et le sexe, cette instabilité constante qui transforme l'encuentro en desencuentro sont le signe de consciences elles aussi éclatées, multiples,

\footnotetext{
2. La même musique est utilisée dans Ana y los lobos, liée au personnage mystique incarné par Fernando Fernán Gómez. Coïncidence ? Résonance, en tout cas.
} 
agrégats aléatoires d'attributs qui se forment au gré des circonstances et des regards d'autrui.

Désorientés, indécis, inquiets voire angoissés, les personnages n'hésitent pas à recourir à la pensée magique -que ce soit la voyance ou ce rituel de santería auquel se livre Jaime dans l'espoir de récupérer Rafael, sans parler du gourou venu reprendre en mains ce dernier. Ce type de comportement, s'il peut se comprendre chez le jeune et «invertébré» Rafa, ne laisse pas de surprendre de la part d'une Angela ou d'un Jaime qui, par ailleurs, manifestent un sens critique, souvent ironique, à l'égard des autres et d'eux-mêmes. Un tel mélange, qui affecte non seulement la micro-société convoquée par le film mais aussi la conscience individuelle, dit à la fois le malaise d'une société en perte de repères et dont les éléments les plus faibles deviennent les proies rêvées de la pensée sectaire, et le manque d'unité d'un sujet contradictoire, instable, à facettes multiples.

La pluralité est également celle des discours et des points de vue, qui intègre le regard allogène (Westphal, 2007: 209)3 ${ }^{3}$-les Argentins à Madrid- ou le regard de celui qui jusqu'à une époque récente n'était que le «regardé», bouleversant ainsi les hiérarchies instituées. Car les regardants, dans le film, sont d'abord les femmes -la sensuelle Rosa, généreuse et inconstante, soumise à ses désirs et fidèle à son amour- et les homosexuels. Le prototype viril -Leopoldo-Tarzanest mis à mal avec humour, l'hétérosexuel traité, avec humour, d' "animal

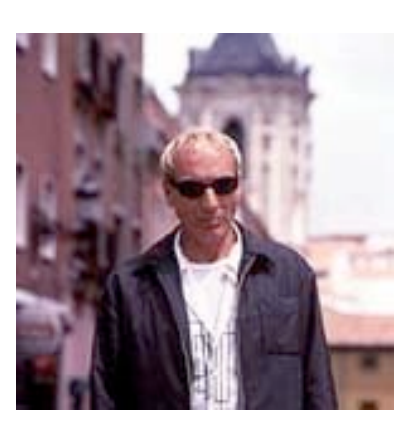
inférieur», l'homosexualité exaltée à travers le personnage séduisant, talentueux, cultivé, drôle de Jaime, incarné avec virtuosité par Eusebio Poncela (on pense à Almodóvar). Par cette «remise en cause de la relation hiérarchique entre la référence centrale (discours dominant) et les manifestations créatives de la marge (discours contre-hégémonique)» (Westphal, 2007: 204), le film de Vicente Molina s'inscrit dans le droit fil d'une esthétique et d'une pensée postmoderne.

\footnotetext{
${ }^{3}$ Bertrand Westphal distingue le point de vue endogène ou autochtone de l'espace, le point de vue exogène qui marque la vision du voyageur, empreint d'exotisme, et le point de vue allogène, situé entre les deux, et qui «est le propre de tous ceux et de toutes celles qui se sont fixés dans un endroit sans que celui-ci leur soit encore familier, sans non plus qu'il demeure pour eux exotique».
} 
Le modèle épistémologique et culturel de la mosaïque semble donc bien régler l'adéquation entre le sujet et la structure filmique. Dällenbach, dans son livre, souligne l'ambivalence d'un tel modèle, apte «à signifier aussi bien un assortiment hétéroclite qu'un ensemble harmonieux» (Dällenbach, 2001: 157). Le tout est de savoir ce qui domine, d'une discontinuité dysphorique qui serait constitutive du monde et de l'être, ou d'une synthèse euphorique de fragments subsumés par un principe de cohérence unitaire. En d'autres termes, existe-t-il dans le sujet un noyau durable qui garantirait son identité ou est-il pure dispersion, constitué d'une succession ininterrompue d'instants changeants? D’un côté, l'idée même de mosaïque renvoie à une démarche esthétisante de totalisation. Le dénouement euphorique du film semble d'ailleurs réduire l'éclatement structurel et la dispersion du sujet par une vraie rencontre. Mais on peut le lire également comme une concession à une convention générique visant à masquer l'évidente discontinuité de nos vies et l'impossible totalisation. Le happy ending permettrait dans ce cas de mettre arbitrairement fin à une ronde qui, sinon, n'aurait aucune raison de jamais s'arrêter. La tonalité aigre-douce, tout ensemble enjouée et mélancolique, ludique et désenchantée du film préserve l'ambivalence. Jusqu'à quel point, d'ailleurs, la discontinuité, aujourd'hui, est-elle ressentie comme une imperfection ? Dans ce sens, on peut penser que le modèle de la mosaïque contemporaine permet au film de «revendiquer (...) le discontinu tout en continuant à se prévaloir de sa légitimité esthétique et de sa vertu consonante» (Dällenbach, 2001: 100). Question de point de vue...

Quoi qu'il en soit, s'il est vrai que Sagitario restitue un état du monde dominé par le principe d'incertitude, il se peut aussi que, dans le même temps, il contribue à en réaménager la forme, obéissant à cette double vocation de la fiction de rendre compte du réel et d'influer sur ses représentations.

\section{Bibliografía}

BRESSON, Robert (1975). Notes sur le cinématographe. Paris: Seuil.

DÄLLENBACH, L. (2001). Mosaïques. Paris: Ed. du Seuil.

ECHENOZ, Jean (1983). Cherokee. Paris: Ed. de Minuit.

WESTPHAL, Bertrand (2007). Géocritique, Paris: Ed. de Minuit. 